\title{
Prediction of Grinding Work Roll Demand in a Job Shop Company By using Artificial Neural Network and ARIMA Method
}

\author{
Yusraini Muharni ${ }^{1 *}$, Ade $\operatorname{Irman}^{1}$, Muhammad Ilhamsyah $^{2}$ \\ ${ }^{1}$ Department of Industrial Engineering, Engineering Faculty, Universitas Sultan Ageng Tirtayasa, \\ Banten, Indonesia 42435 \\ ${ }^{2}$ Production System Laboratory, Department of Industrial Engineering, Engineering Faculty, \\ Universitas Sultan Ageng Tirtayasa, Banten, Indonesia 42435
}

\begin{abstract}
This study concern about forecasting grinding work roll demand in a job shop company located in industrial area in Cilegon. This factory main production is fabrication, which accepts various orders from other companies especially from the company around. Grinding Work Roll is one of those products that frequently request by customer. Although the order is frequent but the volume is fluctuation month by month. This situation drives the company to face the problem in preparing the resources required in fabrication process specially in scheduling the operators. To cope with this problem, we proposed to apply two robust forecasting methods, Artificial Neural Network and ARIMA to help in prediction the grinding work roll demand so as the company could make a good plan for the production process. The best architecture for ANN is obtained through applying Taguchi Method which applies Levenberg-Marquardt algorithm as Training Function. The best number for hidden layer is 10, while Momentum is 0.9 . The Prediction result shows that ANN predicts better than ARIMA Method according to the lower Mean Square Error (MSE). MSE Value for ANN is 0.002 while for ARIMA MSE is 0.0043 . From this study, we experienced that by applying Taguchi method could improve the performance of Artificial Neural Network.
\end{abstract}

\section{Introduction}

The role of forecasting in today's business world is counting essential as company focus on improving customer satisfaction while reducing the cost of fulfill demand and running production process [1]. Forecasting is crucial input to wide range of business decision making process. This research was undergone in a job shop company with fabrication as main activity. Located in industrial area, this company receives various orders mainly from manufacturer nearby. One regular order comes every month from a loyal customer to fabricate Grinding Work Roll. The problem exist in this order is the variation in number of orders that changeability each month so at the time order could be increased while decrease in the next month. This situation influences the completion time of the order, since it over the due date in the contract causing losses for companies and clients. For example is the demand for April 2008 there is a demand of 12 units but the output produced only 10 units then the next month is May 2008 demand decreased

\footnotetext{
${ }^{*}$ Corresponding author: yusmuharni@gmail.com
} 
dramatically to one unit but the output produced exceeds the demand of two units. This situation drove the company into losses because if production exceeds demand. It will still be given to the client because there is no place to accommodate it, whereas if the production does not reach the request it will be fined. Therefore the company intends to predict the number of requests in the Grinding Work Roll for the upcoming year in order to prepare the resources better and avoid further losses in the future.

Time series forecasting method has been known for years such as simple linear regression, exponential smoothing etc. However the simple linear regression required causal variables that explain the observed time series [1]. On the other hand, with only time series data with monthly basis, without any explanatory variables we directed into ARIMA models since it has advantage of determining valuable information from time series. There are three types of model could be applied: (1) Autoregressive (AR) models, (2) Moving Average (MA) Models, (3) Mixed Autoregressive-Moving Average (ARIMA) model. Whereas in fact, fluctuating data is difficult to meet the assumptions that exist in time series analysis as well as regression analysis, so that becomes an obstacle, therefore, an alternative method required which is free from assumptions with good estimation ability, i.e. Non parametric method, one of which can use Artificial Neural Network because this method is able to identify pattern from an input data (input) by using learning method to further trained to study pattern of past data and trying to find function that connect pattern of past data with output that desirable at this time.

Therefore, this study focus on applying Artificial Neural Network and ARIMA method for predicting the number of Grinding Work Roll demand since the data used is fluctuated and has a large scale. However, Artificial Neural Network Method is a more suitable method to use. The objective of this study is the company able to anticipate an increase in the number of orders, and can prepare the resources precisely

\section{Literature Review}

Forecasting has come to play an important role in managing business. To support sales and operations planning process, forecasting can be integrated effectively by involved data from previous sales [2]. The sufficient data in a time series basis can support the time series forecasting process especially for sales forecasting [3].

Forecasting is the activity of predicting or predicting what will happen in the future with a relatively prediction requires accurate data in the past, so that can be seen prospects of future situations and conditions. In general, the use of forecasting is as follows:

1. As a effective and efficient tool in planning.

2. To determine future resource requirements.

3. To make the right decision.

The Usefulness of forecasting is seen in a decision making. A good decision is a decision based on consideration of what will happen at the time of the decision in the various activities of the company. Nevertheless note that the forecast is always an element of error, so that needs to be considered is an attempt to minimize the error of the forecast $[1,5]$. One aspect of making good forecast is deeply understand what comprises the previous data to be used in preparing forecast [5].

Artificial neural networks (ANN) originated from modeling neural networks in the human brain with similar work characteristics with biological neural networks. It can also design the basic structure of a network that is a neuron into a definite design such as pattern recognition faster. This network resembles the human brain in two ways, namely, knowledge acquired by the network through the learning process, and the connection strength between neurons is known as the synaptic weight used to store knowledge [6]. ANN algorithms are commonly used in pattern classification, pattern completion, approach function, prediction, optimization, and control system applications [7]. Artificial neural 
networks is an information processing system that has certain performance characteristics similar to that of human biological neural networks that have been developed as generalizations of mathematical models of human cognition or biological nerves. Generalized mathematics formsANN from biological neural networks, assuming [4]:

1. Information processing occurs in many simple elements (neurons)

2. The signal is sent between the neurons through the link

3. The liason between neurons has a bonded weight which in the network multiplies the transmission signal.

4. Each neuron implements an activation function (usually nonlinear) on its input to determine the output signal.

Forecasting time series data using ARIMA model is encouraging to develop as long data series available (at least 50 data points). The advantages of this model are the ability to handle variability in the data as long as the series has stationary pattern. Besides ARIMA model can be applied for short, medium, and long term forecast horizon. [1]

\section{Research Method}

\section{A. Data}

The forecasting process begins with the collection of historical data on a monthly basis. Data are collected from the year 2008 to 2016 from a job shop company which fabrication as main business.

\section{B. Artificial Neural Network}

In this study, we apply the Artificial Neural Network Model with multilayer architecture consist of three layers: Input layer, Hidden layer, and Output layer as seen in Figure 1.

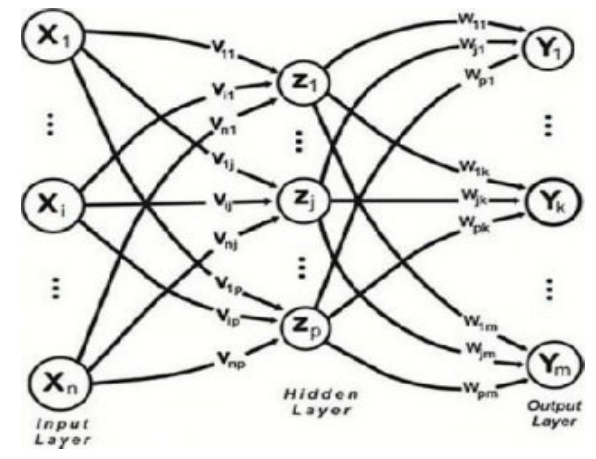

Fig 1. Multilayer Architecture of ANN [4]

We conduct a Taguchi Method in order to select the best ANN Architecture design. Three factors are considered influenced to the accuracy such as learning function, number of hidden layer and the momentum.

\section{Taguchi Method}

First introduced by Genichi Taguchi, this method is recognised as an effective way to conduct experiments since it could improve the efficiency by reducing experimental costs. Originally the method is applied with main focus to improve product quality through designed. Taguchi method introduces orthogonal arrays as a technique to find the best combination of manufacturing factors while reducing 
the experiments $[12,13]$. Taguchi method applies the orthogonal array is noted as $\mathrm{L}_{\mathrm{a}}\left(\mathrm{b}^{\mathrm{c}}\right)$, where $\mathrm{a}$ is the number of factor combination in the experiment, $\mathrm{b}$ is the number of level for factors, and c represents the number of factors in the experiment. The selection the number of factors and the number of levels for each factor in the experiment is based on the proper orthogonal array. In this study, we applied an orthogonal array as $\mathrm{L}_{9}\left(3^{3}\right)$ meaning that we have 9 of factor combinations in the experiment, three level for factors, and three factors in the experiment which are learning function, number of hidden layer and the momentum.

\section{Result And Discussion}

Table 1 is a recapitulation of the best architectural design results. Based on the combination Taguchi method used is the combination of number 3 that is with the training function Levenberg-Marquardt, hidden layer 10 , and momentum of 0.9 . This combination yields an average MSE of 0.00105 .

Table 1. The Recapitulation of ANN's Best Architecture Design with Taguchi Method

\begin{tabular}{cccccccc}
\hline \multirow{2}{*}{ No } & \multirow{2}{*}{ Fungsi Pelatihan } & $\begin{array}{c}\text { Hidden } \\
\text { Layer }\end{array}$ & \multirow{2}{*}{ Momentum } & & \multicolumn{4}{c}{ MSE } & \begin{tabular}{c} 
MSE \\
\cline { 5 - 6 }
\end{tabular} & & Replikasi 1 & Replikasi 2 & Replikasi 3 & Rata-rata \\
\hline 1 & Levenberg-Marquardt & 1 & 0.1 & 0.00113 & 0.00322 & 0.00151 & 0.00195 \\
2 & Levenberg-Marquardt & 5 & 0.5 & 0.00124 & 0.00231 & 0.00157 & 0.00171 \\
3 & Levenberg-Marquardt & 10 & 0.9 & 0.00096 & 0.00099 & 0.00119 & 0.00105 \\
4 & Bayesian Regularization & 1 & 0.5 & 0.00130 & 0.00271 & 0.00151 & 0.00184 \\
5 & Bayesian Regularization & 5 & 0.9 & 0.00115 & 0.00581 & 0.00323 & 0.00340 \\
6 & Bayesian Regularization & 10 & 0.1 & 0.00118 & 0.00121 & 0.00141 & 0.00127 \\
7 & Gradient Descent & 1 & 0.9 & 0.00129 & 0.00235 & 0.00379 & 0.00247 \\
8 & Gradient Descent & 5 & 0.1 & 0.00152 & 0.00967 & 0.00912 & 0.00677 \\
9 & Gradient Descent & 10 & 0.5 & 0.00143 & 0.00371 & 0.00195 & 0.00236 \\
\hline
\end{tabular}

The best performance results of the Levenberg-Marquardt training function can be seen in Figure 2 .

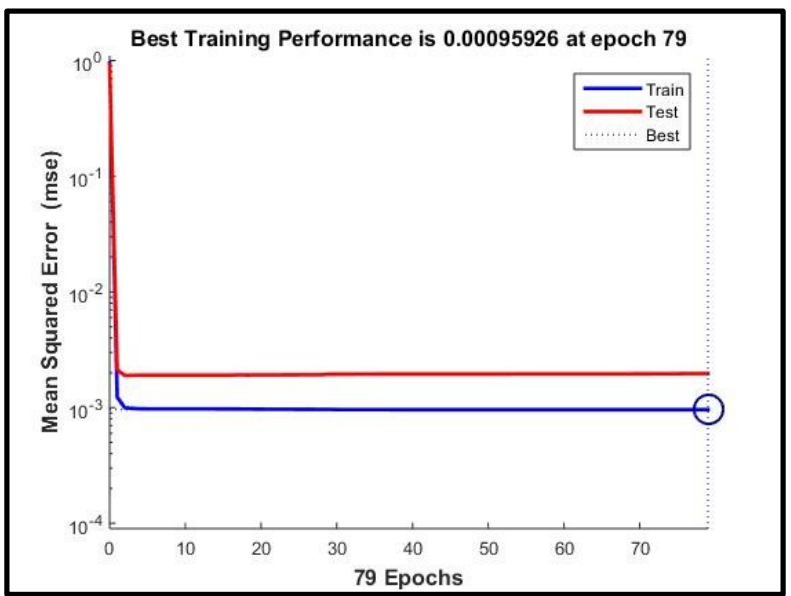

Fig 2. Best Levenberg-Marquardt Performance Result

Figure 2 shows the performance results of the third alternative design with LevenbergMarquardt training function, 10 hidden hidden layers, and Momentum of 0.9. The design produced the best MSE of 0.00095926 when it reached the 79th iteration. Testing process is done by using the best architectural design that has been obtained by using Taguchi 
method, network structure used consists of training function using Levenberg-Marquardt, hidden layer amounted to 10 , and momentum of 0.9 .

Comparison between target and network output aims to see the proximity between data. If getting in between the target and the network output then the difference error is small or not much different between the target with the output network. The regression test graph shows the result of 0.96948 can be seen in Figure 3.

Comparison of ANN forecasting result is done to ascertain whether ANN method is better than other method, for comparison used ARIMA method, based on previous research used ARIMA method $(1,1,0)(2,1,0) 12$. Comparison of ANN method with ARIMA can be seen in Table 2.

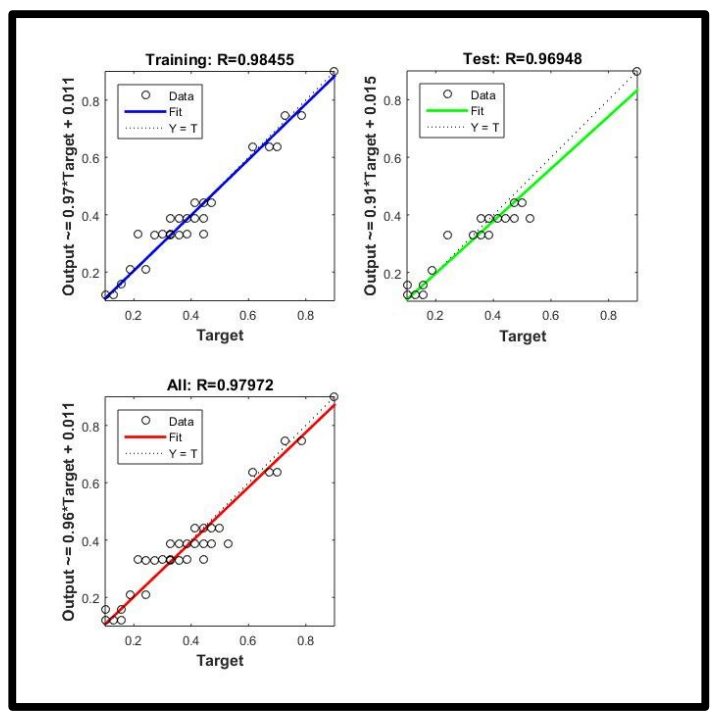

Fig 3. Regression

Table 2. ANN and ARIMA Method Comparison

\begin{tabular}{|l|l|c|}
\hline No & Metode & MSE \\
\hline 1 & Artificial Neural Network & 0.0020 \\
\hline 2 & ARIMA & 0.043 \\
\hline
\end{tabular}

Based on the above table can be seen ANN method has a lower MSE compared with ARIMA method where ANN method has MSE 0.002 and ARIMA method has MSE of 0.043. Thus the best result for this research is using ANN method. Here is the ARIMA and ANN comparison plot to the actual data: 


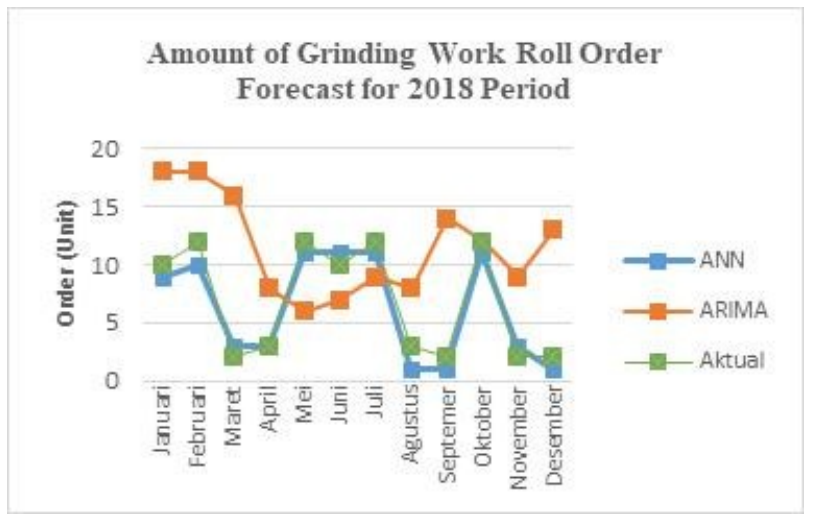

Fig 4. Comparison Between ANN and ARIMA Method Result over Actual Data

Comparison of forecasting the number of order Grinding Work Roll for period 2018 can be seen in Figure 4 that the forecasting with ANN method shows no significant difference to actual data, whereas for forecasting using ARIMA method seen in plot of graph there is a significant difference compared with ANN method. Therefore can be inferred based on MSE, that the ANN method in this work outperform the ARIMA method.

\section{Conclusion}

Based on the results of research, it can be drawn conclusions, as follows:

1. The best Artificial Neural Network architecture design for demand for Grinding Work Roll is to use Levenberg-Marquardt training function, 10 hidden layers, and 0.9 momentum.

2. The results of the demand forecast for Grinding Work Roll using Artificial Neural Network for the period of 2018 are 9 units, 10 units, 3 units, 3 units, 11 units, 11 units, 11 units, 1 unit, 1 unit, 11 units, 3 units, and 1 unit.

3. Forecasting result using ANN method produce MSE equal to 0.0019668 while for ARIMA method produce MSE equal to 0.0043. Based on the comparison of MSE values of ANN method is outperform the ARIMA method.

4. The suggestion for further research is to conduct further research on forecasting production demand on other products as well as the addition of influencing factors to determine the best network architecture to produce more accurate forecasting, resulting in more precise forecasting results for subsequent production.

\section{References}

1. J. H. Wilson, B. Keating, J.G. Solution Inc, Business Forecasting With ForecastXTM, Mc Graw Hill, Singapore (2009)

2. T. Alhadeff, J. of B.. Forecasting 23.1. (2004)

3. S. Harris, J. of B.. Forecasting (1995-96)

4. Fausett, L. Fundamental of neural Network : Architecture. Algorithms and Applications. Prentice Hall Inc., New Jersey. (1994)

5. M.A. Moon, T. Mentzer, J. of B. Forecasting.18.2.(1999)

6. J. Lawrence, Introduction to neural networks. Design, theory, and applications. Nevada City, CA: California Scientific Software; 1994. 
7. L. Medsker, J. Liebowitz, Design and development of expert systems and neural networks. New York: Macmillan College Publishing Company; 1994.

8. L.Wang, Z.-G. Wang, H. Qu, S.Liu. Appl. Soft Computing. (2018) https://doi.org/10.1016/j.asoc.2018.02.004

9. D. Matsypura, R. Thompson, A.L. Vasnev. Omega. (2017). Doi:10.1016/j.omega.2017.06.010

10. D. Nibbering, R. Paap, M.V.D. Wel. Int. J. of Forecasting 3 (2018)

11. S. Benabderrahmane, N. Melloouli., M. Lamolle. Knowledge-Based Systems 000. 1-19 (2018)

12. G. Tkacz. Int. J. of. Forecasting. 17.1.(2001)

13. M.-L. Huang, Y.-H. Hung, Z.-S. Yang, Measurement. 54.(2016)

14. G. Taguchi, E.A. Elsayed, T.C. Hsiang, Quality Engineering in Production System, Mc Graw Hill Book company (1989)

15. K. Ouyang, H.-W. Wu, S.-C. Huang, S.-J. Wu. Energy (2017) 